\title{
Clinical Course of Bark Scorpion Envenomation Managed Without Antivenom
}

\author{
Ayrn O'Connor • Anne-Michelle Ruha
}

Published online: 5 May 2012

(C) American College of Medical Toxicology 2012

\begin{abstract}
Bark scorpion envenomation is potentially life threatening in children and traditionally treated with antivenom (AV). We sought to describe the clinical course, management, complications and outcome of children with severe scorpion envenomation treated with supportive care during a period when $\mathrm{AV}$ was unavailable. A retrospective chart review was performed, all children presenting to a referral hospital between September 1, 2004 and July 31, 2006 with severe scorpion envenomation not receiving AV, were included. A standardized data abstraction form was used to record time of symptom onset, time to healthcare facility (HCF), clinical findings, treatment, complications, and length of stay. Eighty-eight patients were included with mean age of 3.7 years $(0.33-12)$. Mean time to symptom onset was $20 \mathrm{~min}(0-130)$ and mean time to HCF was 79 min (10-240). Incidence of clinical manifestations include: neuromuscular agitation, $100 \%$; opsoclonus, $97 \%$; hypersalivation, $81 \%$; tachycardia, $82 \%$; hypertension, $49 \%$; vomiting, $38 \%$; fever, $28 \%$; respiratory distress, $33 \%$; and hypoxia, $18 \%$. Complications included rhabdomyolysis in $18(20 \%)$ and aspiration in $12(13 \%)$ patients. Intubation was required in $24 \%$ of patients. The most frequently used agents to control symptoms were benzodiazepines $(98 \%)$ followed by opioids $(69 \%)$. Intravenous fluids were given to $84 \%$. Mean length of stay was $29 \mathrm{~h}$ (range, 6-73 h). There were no deaths. In addition to the classic findings of neuromuscular hyperactivity, opsoclonus, and hypersalivation, a high incidence of hyperadrenergic findings and respiratory compromise are noted in this series.
\end{abstract}

A. O'Connor $(\bowtie) \cdot$ A.-M. Ruha

Banner Good Samaritan Medical Center,

925 E. McDowell Road, 2nd Floor,

Phoenix, AZ 85006, USA

e-mail: Ayrn.OConnor@bannerhealth.com
A significant number of patients required mechanical ventilation. Benzodiazpines and opioids were the most common medications used to control symptoms.

Keywords Scorpion envenomation · Centruroides sculpturatus $\cdot$ Respiratory failure $\cdot$ Motor hyperactivity Hyperadrenergic effects

\section{Introduction}

Centruroides sculpturatus envenomation was once responsible for the most venom-related fatalities in Arizona [1]. Today, a sting by this species, known also as the bark scorpion, is rarely fatal. Mortality has declined presumably due to both improved access to healthcare facilities and advancements in supportive care. Envenomation remains a significant public health concern in the desert southwest, particularly for young children. In 2010, there were 3,498 visits to emergency departments in Arizona due to scorpion stings, with the highest rate of visits occurring in young children [2].

Historically, a whole immunoglobulin $\mathrm{G}(\mathrm{IgG})$ antivenom derived from goat serum was manufactured at the Arizona State University Antivenom Production Laboratory and was available only in the state of Arizona [3]. This antivenom was used in emergency departments throughout much of the state, rapidly reversing clinical effects and typically avoiding hospital admission [4]. However, its use was associated with a significant incidence of anaphylaxis and delayed hypersensitivity. As a result some clinicians chose not to use it and relied solely on supportive care [5, 6]. In 2001, the manufacture of whole IgG antivenom ceased and by 2004 all supplies were exhausted [4]. At this time, supportive care became the only treatment option, outside of enrollment in a clinical trial of $\mathrm{Fab}_{2}$ antivenom. 
Reports in the modern medical literature describing the clinical course and complications associated with severe scorpion envenomation, as well as supportive care and interventions used in victims, are scarce. We describe the largest series of patients reported in the medical literature with bark scorpion envenomation treated without antivenom.

\section{Methods}

Poison center records and patient log books kept by the Department of Medical Toxicology were reviewed to identify patients with scorpion envenomation who presented to Phoenix Children's Hospital (PCH) between September 1, 2004 and June 30, 2006. The patients represent those who were cared for by a medical toxicologist, which was the common practice for severe envenomations. The study dates were chosen to include the period of time immediately following the exhaustion of all supplies of goat-derived scorpion antivenom and prior to participation in a clinical trial of a $\mathrm{Fab}_{2}$ antivenom. The study was approved by the PCH Investigational Review Board.

All patients meeting criteria for grades III or IV scorpion envenomation were included in the study. (see Table 1 for grading system). Two investigators used a standardized data abstraction form to record data from poison center and hospital records for each subject. Poison center records were included in order to capture information from the prehospital setting. Data included time to symptom onset, time to healthcare facility (HCF), vital signs, symptoms, peak serum creatinine, peak serum creatinine phosphokinase (CPK), chest radiograph (CXR), treatments, and length of hospital stay. Data was entered into a Microsoft Excel spreadsheet and descriptive statistical analysis was performed.

A list of definitions were created a priori, agreed upon by the investigators and included the following: rhabdomyolysis, $\mathrm{CK}>1000 \mathrm{IU} / \mathrm{L}$; fever, temperature $>38^{\circ} \mathrm{C}$; hypoxia, oxygen saturation $<94 \%$; aspiration pneumonia, infiltrate on CXR. Age-appropriate vital signs from the National

Table 1 Signs and symptoms of scorpion envenomation

\begin{tabular}{ll}
\hline Grade & Description \\
\hline I & $\begin{array}{c}\text { Localized pain and/or paresthesias at the site of } \\
\text { envenomation }\end{array}$ \\
II & $\begin{array}{l}\text { Pain and/or paraesthesias remote from site of envenomation } \\
\text { III } \quad \text { Either cranial nerve or neuromuscular dysfunction } \\
\\
\text { Cranial nerve dysfunction: tongue fasiculations, } \\
\text { hypersalivation, slurred speech or opsoclonus } \\
\text { Neuromuscular dysfunction: involuntary shaking and } \\
\text { jerking of the extremities }\end{array}$ \\
IV $\quad$ Both cranial nerve and neuromuscular dysfunction
\end{tabular}

Adapted from grading system outlined in Curry et al. [8]
Institute of Health (NIH) Clinical Center were utilized to determine presence of hypertension and tachycardia [7]. If systolic blood pressure exceeded the age-based 99th percentile and height-based 95th percentile, the patient was considered hypertensive. Tachycardia was established if heart rate exceeded the upper limit of the age-based normal range as delineated by the NIH [7].

\section{Results}

Ninety-one patients were identified; three patients were excluded for the following: one received investigational $\mathrm{Fab}_{2}$ antivenom at the referring hospital, one was not believed to be a scorpion envenomation, and no chart could be identified for one of the patients. Consequently, 88 met inclusion criteria. The mean age was 3.7 years (range, 4 months-12 year). Figure 1 describes the age distribution of patients. The mean time to symptom onset was $20 \mathrm{~min}$ (range, 0-130 $\mathrm{min}$ ). The mean time to HCF was $79 \mathrm{~min}$ (range, 10-240 $\mathrm{min}$ ) and mean length of stay was $28.7 \mathrm{~h}$ (range, 2-69 h).

Clinical manifestations are summarized in Table 2. Agitation and motor hyperactivity were universally present. Opsoclonus, or rapid irregular multidirectional dysconjugate eye movement, was the second most common finding, noted in $96 \%$ of patients. Tachycardia (82\%) and hypersalivation (81\%) were also prominent clinical findings. Hypertension was documented in nearly half of the patients. Vomiting was noted in $38 \%$ of patients and typically occurred early and was transient, with the majority of episodes $(25 / 33)$ described in the prehospital setting. Fever was also frequently noted.

Respiratory distress occurred in one third of patients, with stridor described in 15 of 88 patients $(17 \%)$ and transient hypoxemia in 16 of 88 patients (18\%). Respiratory compromise resulted in intubation and mechanical ventilation in $24 \%(21 / 88)$ of patients.

\section{Age Distribution}

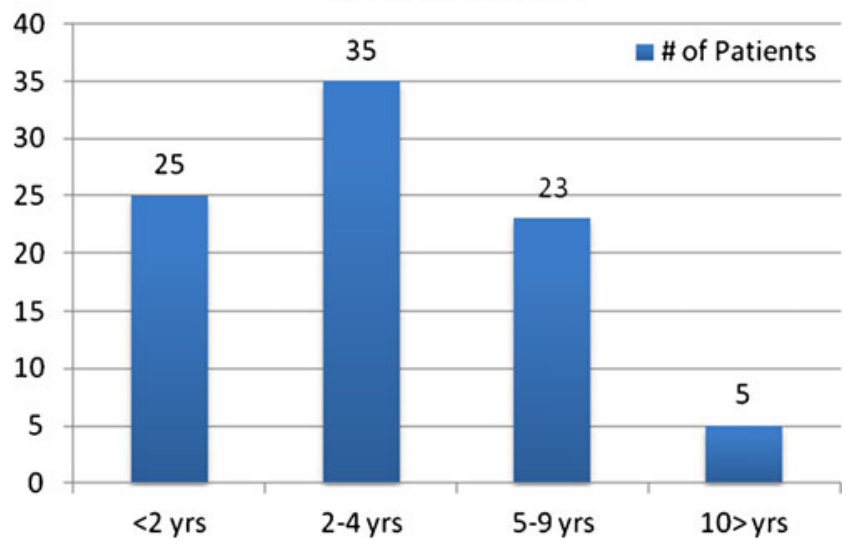

Fig. 1 Age distribution of patients 
Table 2 Occurrence of clinical manifestations

\begin{tabular}{ll}
\hline Clinical sign & $\begin{array}{l}\text { Percent of } \\
\text { patients }\end{array}$ \\
\hline Motor hyperactivity & 100 \\
Opsoclonus & 96 \\
Tachycardia & 82 \\
Hypersalivation & 81 \\
Hypertension & 49 \\
Vomiting & 38 \\
Respiratory distress & 33 \\
Fever & 28 \\
Hypoxemia & 18 \\
Stridor & 17 \\
Diaphoresis & 11 \\
\hline
\end{tabular}

Treatment involved administration of benzodiazepines in the vast majority (98\%) of patients, as well as intravenous fluids in $84 \%$, and opioids in $69 \%$. The particular benzodiazepine used varied, although midazolam and lorazepam were utilized most often. Fentanyl was the most common opioid analgesic used. Other common therapies included acetaminophen (32\%), atropine (30\%), and diphenhydramine $(25 \%)$. Nonsteroidal anti-inflammatory drugs were administered to $11 \%$ of patients (10 of 88 ). Interestingly, two patients received chloral hydrate, two received pentobarbital, and two were given haloperidol to control agitation and motor hyperactivity prior to transfer or consultation with toxicology service. Additional treatments included racemic epinephrine nebulizers in $13 \%(11 / 88)$ of patients and steroids in $17 \%(15 / 88)$. Two patients were given subcutaneous epinephrine and eight (9\%) patients received antibiotics.

Creatinine phosphokinase was measured in $69 \%(61 / 88)$ of patients, with $20 \%(18 / 88)$ developing rhabdomyolysis defined as $\mathrm{CK}>1000 \mathrm{IU} / \mathrm{L}$. No patients developed a rise in creatinine as a consequence of rhabdomyolysis. A CXR was obtained in $33 \%(29 / 88)$ of patients, with aspiration confirmed by the presence of an infiltrate in $14 \%(12 / 88)$. Complications are summarized in Table 3. No mortality occurred.

\section{Discussion}

C. sculpturatus is the only scorpion that produces clinically significant toxicity in the USA and is endemic to the southwest. In recent decades, hospital admission following scorpion sting was infrequent throughout most of Arizona due to use of an anti-Centruroides whole $\mathrm{IgG}$ antivenom, but after this antivenom became unavailable in 2004, intensive care admissions for scorpion envenomation dramatically increased, with one report citing a nearly $500 \%$ increase in admissions over baseline [4].
Table 3 Summary of clinical complications

\begin{tabular}{lll}
\hline Complication & $\begin{array}{l}\text { Number of } \\
\text { patients }\end{array}$ & $\begin{array}{l}\text { Percent of total patients } \\
\text { (percent of patients tested) }\end{array}$ \\
\hline $\begin{array}{l}\text { Rhabdomyolysis } \\
\begin{array}{c}\text { Respiratory failure } \\
\text { and intubation } \\
\text { aspiration pneumonia }\end{array}\end{array}$ & 18 & $20(30)$ \\
\hline
\end{tabular}

Venom from $C$. sculpturatus alters sodium channel inactivation and lowers the threshold for membrane depolarization, producing prolonged action potentials and repetitive axonal firing $[8,9]$. The resultant clinical manifestations that are commonly described include both painful paresthesias that may be localized to or progress beyond the sting site, and cranial nerve and somatic motor abnormalities. Motor hyperactivity is characterized by uncontrolled excessive jerking of the extremities, twisting and arching of the torso, and inability to lie still. Cranial nerve dysfunction manifests as opsoclonus, tongue fasciculations, loss of pharyngeal muscle control with difficulty swallowing, and stridor. These effects, coupled with hypersalivation, can threaten airway integrity. Pulmonary edema may also rarely occur as a direct effect of envenomation [10]. Children are at greatest risk for the most severe manifestations of envenomation $[8,10,11]$.

It is well known that infants and toddlers are at increased risk for morbidity following a bark scorpion sting. An interesting finding in this study was the distribution of severe illness within the pediatric age groups. Nearly $72 \%(63 / 88)$ of the patients were older than 2 years of age (Fig. 1). Also, of the 21 patients with respiratory failure requiring intubation, ten were 2 years of age or older, and two were 8 years old. These findings highlight the fact that even older children are at risk for severe envenomation and airway compromise.

This study also reveals the incidence of multiple clinical findings that occur in association with severe bark scorpion envenomation in children. By abstracting information obtained in the prehospital setting, as well as the emergency department and intensive care unit, we are able to illustrate the clinical course of scorpion envenomation, from vomiting that occurs at the onset of toxicity to respiratory failure that develops in a significant proportion of patients. The vast majority of our patients exhibited the expected neuromuscular agitation and cranial nerve findings (defining a grade IV envenomation). Adrenergic findings were also significant, with tachycardia present in the majority of patients, and hypertension and fever also common. Adrenergic symptoms responded to sedatives and analgesics in all cases, without need for antihypertensive or antiarrhythmic medications.

Vomiting was documented in over one third of patients. Vomiting was generally transient and occurred early, often prior to presentation to a healthcare facility. While this finding is not often noted in emergency department patients 
who present with scorpion envenomation, it can be a useful historical piece of information to aid the physician in establishing a diagnosis of scorpion sting.

The supportive care of patients with bark scorpion sting has previously been described as use of benzodiazepine monotherapy [12]. Our experience treating adults and older children with grades III and IV envenomations, revealed that paresthesias are painful and unrelieved by administration of benzodiazepines alone. For this reason, we strongly advocate use of opioid analgesics to control pain as well as benzodiazepines for agitation. The opioid analgesic most commonly used in this study was fentanyl and is a reflection of the local practice preference due to its short duration of action, ease of titration, and reduced histamine release in comparison to other opioids. Respiratory failure may result from envenomation even prior to treatment, and these classes of medications add to the risk of airway loss, so doses are titrated carefully with close observation and intervention as needed. Patients often require very large doses of sedative hypnotic medications to control symptoms [11, 12], and in the most severe cases neuromuscular paralysis with sedation and mechanical intervention is necessary $[10,12]$.

Atropine was administered to a significant proportion of patients in this study. This anticholinergic medication is typically administered in small doses to dry secretions in patients with significant hypersalivation [13]. There are no studies to establish its efficacy in this setting and excessive doses should be avoided to prevent anticholinergic toxicity.

Many patients were treated with diphenhydramine and several with steroids. Many parents and some prehospital providers mistake neurotoxic effects of scorpion envenomation as an allergic reaction to the arthropod. It is not uncommon for patients to receive treatments targeted at an allergic reaction, including epinephrine, in the prehospital setting as a result of this misconception. Considering the adrenergic effects of scorpion envenomation, administration of epinephrine is especially dangerous. Similarly, racemic epinephrine is not indicated to treat stridor in this setting since stridor is not caused by airway inflammation.

Some of the medications administered to patients in this series were given prior to diagnosis of bark scorpion envenomation. A scorpion is often not found and the child is unable to provide history; therefore, patients may receive antibiotics and other therapies as their initial evaluation proceeds. We do not recommend use of antibiotics, haloperidol, or antihistamines to treat the effects of scorpion envenomation.

Limitations of this study include those inherent to the retrospective design. The presence of some clinical effects may not have been documented in all patients and as a result may have been reported as occurring less frequently than they did. Additionally, presence of rhabdomyolysis and aspiration pneumonia could only be determined in those patients with documented CPK and CXR. We were also unable to determine time to resolution of symptoms since this was rarely documented. Instead we recorded length of stay, but this parameter is affected by external factors including physician arrival time for discharging the patient and social issues. The risk of introduction of bias was limited as data was categorical and not subject to reviewer interpretation. Furthermore, the objective of this study was merely descriptive and does not allow for comparison of treatment efficacy or outcomes.

In August of 2011, a Fab 2 anti-Centruroides antivenom (Anascorp ${ }^{\circledR}$ ) was approved by the Food and Drug Administration for treatment of clinically significant bark scorpion envenomations. Unfortunately, the antivenom is extremely expensive, with a single treatment course costing a hospital between $\$ 11,250$ and $\$ 18,750$ [14]. Charges to patients are even higher. This high price has already factored into treatment decisions and limits the use of antivenom in many hospitals in Arizona, highlighting the continued importance of supportive care for treatment of severe scorpion envenomation.

\section{Conclusion}

Bark scorpion sting may produce life-threatening envenomation in young children, with over $20 \%$ of patients in this series intubated for respiratory failure. In addition to the classic findings of neuromuscular hyperactivity, opsoclonus, and hypersalivation, we also noted a high incidence of adrenergic findings, including tachycardia, hypertension, and fever. Treatment with benzodiazepines, opioids, and mechanical ventilation when needed, appears to be effective in managing symptoms of bark scorpion envenomation as an alternative to antivenom.

Previous Presentation of Data The results of this study were presented in abstract form and as a platform during the 2010 NACCT national meeting.

Funding There were no external sources of funding required for this project.

Conflict of Interests Neither author has any conflicts related to this manuscript. Both authors participated in a clinical study in which patients were enrolled and received Anascorp antivenom. 


\section{References}

1. Stahnke HL (1950) The Arizona scorpion problem. J Ariz Med Assoc 7(3):23-29

2. Shacter A. Injuries due to venomous insects and plants among Arizona residents, 2010. Arizona Department of Health Services. September 2011

3. Gateau T, Bloom M, Clark R (1994) Response to specific Centruroiodes sculpturatus antivenom in 151 cases of scorpion stings. Clin Toxicol 32(2):165-171

4. Riley BD, LoVecchio F, Pizon AF (2006) Lack of scorpion antivenom leads to increased pediatric ICU admissions. Ann Emerg Med 47(4):398-399

5. Bond GR (1992) Antivenin administration for Centruroides scorpion sting: risks and benefits. Ann Emerg Med 21(7):788-791

6. LoVecchio F, Welch S, Klemens J et al (1999) Incidence of immediate and delayed hypersensitivity to Centruroides antivenom. Ann Emerg Med 34:615-619

7. National Institute of Health (NIH) Clinical Center. Accessed from: http://www.cc.nih.gov/ccc/pedweb/pedsstaff/age.html. Accessed on 31 Jan 2012
8. Curry SC, Vance MV, Ryan PH et al (1984) Envenomation by the scorpion Centruroides sculpturatus. J Toxicol Clin Toxicol 21:417-449

9. Rodriguez de la Vega RC, Possani LD (2005) Overview of scorpion toxins specific for $\mathrm{Na}$ channels and related peptides: biodiversity, structure-function relationships and evolution. Toxicon 46:831-844

10. Berg RE, Trantino MD (1991) Envenomation by the scorpion Centruroides exilicauda: severe and unusual manifestations. Pediatrics 87(6):930-933

11. Boyer LV, Theodorou AA, Berg RA et al (2009) Antivenom for critically ill children with neurotoxicity from scorpion stings. $\mathrm{N}$ Engl J Med 360:2090-2098

12. Gibly R, Williams M, Walter FG et al (1999) Continuous intravenous midazolam infusion for the Centrutoides exilicauda scorpion envenomation. Ann Emerg Med 34:620-625

13. Suchard JR, Hilder R (2001) Atropine use in Centruroides scorpion envenomation. J Toxicol Clin Toxicol 39(6):595-598

14. Alltucker K. Scorpion antivenom has stinging cost. Nov 13 2011. Accessed from: http://www.azcentral.com/business/ articles/2011/11/10/20111110scorpion-drug-cost.html. Accessed on 26 Jan 2012 